\title{
Estresse e Estratégias de Enfrentamento em Profissionais de Abrigos Institucionais
}

\author{
Rosângela Maria Negri Ferrão Pagnota ${ }^{1}$ \\ Helena Bazanelli Prebianchi ${ }^{1}$ \\ ${ }^{1}$ Pontifícia Universidade Católica de Campinas, SP, Brasil. 'Pontifícia Universidade Católica de Campinas, SP, Brasil.
}

\begin{abstract}
Resumo: O estudo objetivou verificar e analisar os níveis de estresse percebido e as estratégias de copingdos profissionais de abrigos institucionais. Trata-se de um estudo empírico, descritivo e correlacional, com amostra intencional de 45 profissionais de contato direto e indireto com abrigados, em quatro abrigos institucionais. Utilizaram-se quatro instrumentos: ficha de caracterização do abrigo institucional, ficha de caracterização do participante, Impact of Event Scale (IES) e Cope Breve. Para análise dos resultados, foi utilizada a estatística descritiva: valores máximo e mínimo das pontuações nas subescalas de coping, e inferencial: análise de regressão linear e não paramétrico - correlação de Spearman, considerando a natureza e a distribuição dos escores. Os instrumentos IES e Cope Breve foram aplicados e analisados de acordo com as instruções das adaptações adotadas para este estudo. Os resultados demonstraram que os níveis de estresse percebidos foram maiores nos participantes de abrigos institucionais filantrópicos de contato direto com abrigados. A estratégia de coping mais utilizada foi o coping focalizado no problema em ambos os abrigos, e a estratégia de coping com maior correlação com o estresse foi o coping focalizado na emoção e desadaptativo. As variáveis preditoras de estresse foram: tipo de abrigo, a jornada de trabalho, a escolaridade e o coping focalizado na emoção e desadaptativo. Conclui-se que somente a avaliação da saúde dos profissionais de abrigos poderá gerar ações e políticas adequadas a eles dirigidas e detectar as necessidades de aprimoramento e programas de capacitação profissional, contribuindo na qualidade da assistência às crianças e adolescentes abrigados.
\end{abstract}

Palavras-chave: Abrigo, Estresse, Coping, Adaptação Psicológica.

\section{Stress and Coping Strategies in Shelter Professionals}

\begin{abstract}
This study aimed to verify and analyze perceived stress and coping strategies in professionals from institutional shelters. This is a descriptive correlational empirical study conducted with a purposeful sample of 45 professionals from four institutional shelters who were in direct and indirect contact with sheltered individuals. Data were collected using four instruments: institutional shelter characterization form; participant characterization form; the Impact of Event Scale (IES); and the Brief-COPE. Results were analyzed using descriptive (maximum and minimum values of coping subscales) and inferential statistics (linear and non-parametric regression analysis - Spearman's correlation, considering the nature and distribution of the scores). Both the IES and Brief-COPE instruments were applied according to the instructions of the adaptations adopted for this study. Professionals from philanthropic institutional shelters in direct contact with sheltered individuals showed higher levels of perceived stress. In both shelters, the most used coping strategy was problem-focused coping, whereas emotion- and maladaptation-focused coping showed the highest correlation with stress. Type of shelter, working day, education level, and emotion- and maladaptive-focused coping were stress predictors. Our results indicate that developing appropriate actions and policies aimed at
\end{abstract}


shelter professionals require the assessment of their health, enabling authorities to detect the needs for improvement and professional training programs, contributing to the quality of care for sheltered children and adolescents.

Keywords: Shelter, Stress, Coping, Adaptation Psychological.

\title{
Estrés y Estrategias de Enfrentamiento de Profesionales de Refugios Institucionales
}

\begin{abstract}
Resumen: Este estudio propone identificar y analizar los niveles de estrés y las estrategias de coping de los profesionales de refugios institucionales. Este es un estudio empírico, descriptivo y correlacional, con muestra intencional de 45 profesionales de contacto directo e indirecto con los refugiados, en cuatro refugios institucionales. Se utilizaron cuatro instrumentos: Ficha de caracterización del refugio institucional, Ficha de caracterización del participante, Impact of Event Scale (IES) y Cope Breve. Para el análisis de los resultados, se utilizó la estadística descriptiva: valores máximo y mínimo de las puntuaciones en las subescalas de coping, y también inferencial: análisis de regresión lineal y no paramétrico, correlación de Spearman, considerando la naturaleza y la distribución de los puntajes. Los instrumentos IES y Cope Breve fueron aplicados y analizados bajo las instrucciones de las adaptaciones adoptadas para este estudio. Los resultados demostraron que los niveles de estrés fueron más elevados en los profesionales de refugios institucionales filantrópicos de contacto directo con los refugiados. La estrategia de coping más utilizada fue el coping enfocado en el problema en ambos refugios, y la estrategia de coping con mayor correlación con el estrés fue el coping enfocado en la emoción y desadaptativo. Las variables predictoras de estrés fueron el tipo de refugio, la jornada de trabajo, la escolaridad y el coping enfocado en la emoción y desadaptativo. Se concluye que la evaluación de la salud de los profesionales de refugios podrá generar acciones y políticas adecuadas, además de identificar las necesidades de mejora y programas de capacitación profesional, contribuyendo a la calidad de la asistencia a los niños y adolescentes en refugios.
\end{abstract}

Palabras clave: Refugio, Estrés, Coping, Adaptación Psicológica.

\section{Introdução}

$\mathrm{O}$ abrigamento institucional é a sétima medida protetiva a ser tomada em casos de crianças e adolescentes em situação de vulnerabilidade. Descrita como excepcional e provisória, deve garantir condições aos abrigados em termos de segurança, cuidados e possibilidade de reinserção em suas famílias de origem ou, quando se esgotarem todas as tentativas desse retorno, serem encaminhados para uma família extensa ou substituta (Lei no 12.010, 2009).

$\mathrm{O}$ afastamento das crianças e adolescentes de suas famílias de origem desencadeia vários acontecimentos que influenciarão, em menor ou maior grau, suas vidas. Para garantir os direitos fundamentais de crianças e adolescentes, em 2009 o Conselho Nacional dos Direitos da Criança e do Adolescente (Conanda) e o Conselho Nacional de Assistência Social (CNAS) aprovaram a Tipificação Nacional de Serviços Socioassistenciais, por meio da Resolução no 109. A Resolução organizou níveis de complexidade do Serviço Único de Assistência Social (Suas). Os abrigos institucionais estão enquadrados no Serviço de Proteção Social Especial de Alta Complexidade, cuja organização, diretrizes e objetivos são baseados no Estatuto da Criança e do Adolescente - ECA (Brasil, 1990) e nas Orientações técnicas: Serviços de acolhimento para crianças e adolescentes (Brasil, 2009b).

Diversos profissionais buscam assegurar que crianças e adolescentes em situação de abrigamento tenham suas necessidades e seus direitos garantidos, participando diretamente no processo. Ao chegarem às instituições, aos abrigos ou às casas provisórias, 
os abrigados devem encontrar suporte psicológico, físico e educacional para auxiliar na compreensão do que está ocorrendo com eles e suas famílias. É importante que os profissionais, nos abrigos, procurem manter uma postura ética e profissional, atendendo às necessidades de cada criança e adolescente individualmente, pois os abrigados podem buscar vínculos e referenciais alternativos na equipe profissional, tomando-os como "pais substitutos" dentro da instituição, almejando apoio social ou disputando a atenção do profissional com outros abrigados, como ocorreria em suas famílias de origem (Siqueira, Betts, \& Dell'Aglio, 2006).

Os profissionais, ao buscarem o bem-estar dos abrigados, mobilizam-se internamente para lidar com cada caso e, ao mesmo tempo, lidam com as exigências pessoais e familiares de todos os dias que também necessitam de atenção. Nesse sentido, Barros (2014) e Guará (2010) destacam que a falta de incentivos e apoio oferecidos aos profissionais dos abrigos em sua prática e desenvolvimento profissional podem causar desequilíbrio biopsicossocial e levar ao adoecimento, e alertam para o cuidado com o cuidador, já que estes estão expostos constantemente a diversos eventos estressores diariamente.

O estresse é um dos produtos desse desequilíbrio. Ele ocorre quando o indivíduo se defronta com determinada situação para a qual não dispõe de habilidades para lidar, o que produz efeitos negativos, desequilibrando todo o organismo (Lipp, 2000; Selye, 1956). Os eventos estressores podem ter duas origens: a) interna - a maneira de reagir à vida, o modo de ser, o tipo de personalidade; ou b) externa - as exigências da família, do trabalho, do dia a dia, convívio social (Lipp, 2000, 2002).

Lipp (2000) e Silva e Enumo (2014) argumentam que as respostas ao estresse podem ocorrer nos níveis cognitivo, comportamental e fisiológico, sendo que, neste último, quando ativado intensa e frequentemente, esgota os recursos do organismo, levando ao aparecimento de transtornos psicofisiológicos. Essas alterações ocorrem a partir do evento estressor e a percepção, a análise e a interação com o ambiente poderão fornecer subsídios para o indivíduo enfrentar ou não o processo de estresse. O conjunto de esforços utilizados para lidar com o estresse é denominado estratégias de coping.

Segundo Simões (2012), o mecanismo de coping serve como uma proteção para as ameaças ao bem-estar do organismo, e sua eficácia está na capacidade de reduzir o desconforto e evitar o mesmo futuramente. Lazarus e Folkman (1984) estabelecem que as estratégias de coping operam no campo da consciência, podendo ou não ser aprendidas. São centradas em duas categorias: a) no problema: voltada para diminuir o problema, manipulando o evento estressor para removê-lo; b) na emoção: voltada para reduzir as consequências emocionais decorrentes da experiência estressora, buscando adaptação do estado emocional. São consideradas respostas para situações estressantes as seguintes estratégias de coping: autocontrole, confronto, resolução planejada, procura de suporte social, aceitação das responsabilidades, reavaliação positiva da situação, distanciamento do estressor e evitamento/fuga.

A função de "cuidar" pode ser considerada um estressor, posto que o excesso de tarefas, responsabilidades, preocupações, sobrecarga emocional, a precariedade na infraestrutura e nas resoluções de problemas podem levar a um desgaste físico e psíquico em pessoas que realizam essa atividade formal ou informalmente (Lima, 2012). Tendo isso em consideração, este estudo objetivou verificar e analisar os níveis de estresse percebido e as estratégias de coping utilizadas por profissionais das equipes de abrigos institucionais infantojuvenis e identificar os fatores preditores de estresse.

\section{Método}

Trata-se de um estudo descritivo e correlacional, realizado em quatro abrigos institucionais infanto-juvenis, após a aprovação pelo Comitê de Ética em Pesquisa com Seres Humanos da Pontifícia Universidade Católica de Campinas (SP), sob parecer no 2.129.742.

Os dados foram coletados individualmente em salas cedidas pelas instituições, as quais ofereciam condições de privacidade para que os participantes que aceitaram participar da pesquisa respondessem aos instrumentos individualmente, em horários pré-agendados. Os critérios de inclusão utilizados para os abrigos foram: a) acolher crianças e adolescentes em situação de vulnerabilidade retirados de suas famílias de origem; b) histórico de funcionamento de no mínimo dois anos, em situação regular, de acordo com as leis vigentes do município (Alvará); c) respeito às normas estabelecidas pelo ECA e pela 
Resolução no 130 de 2006 do Conselho Nacional de Assistência Social, em relação à equipe profissional e ao espaço físico; e d) assinatura do Termo de Consentimento Institucional. Adotou-se como critério de exclusão o não cumprimento dos critérios de inclusão mencionados.

Os critérios aplicados para inclusão dos profissionais participantes foram: a) fazer parte da equipe profissional no mínimo há três meses; e b) ter assinado o Termo de Consentimento Livre e Esclarecido. Os critérios para exclusão foram: a) ser voluntário ou colaborador externo; e b) ser profissional do Sistema de Garantia de Direitos.

O desenvolvimento deste estudo atendeu eticamente todas as normas nacionais e internacionais para pesquisas envolvendo seres humanos.

\section{Processo de composição da amostra}

A coleta de dados foi realizada em quatro abrigos institucionais infanto-juvenis, sendo dois filantrópicos e duas Organizações da Sociedade Civil de Interesse Público - Oscips (Brasil, 1999) na Região Metropolitana de Campinas (SP), constituída por 20 municípios (Lei Complementar Estadual no 870, 2000).

É necessário esclarecer as diferenças entre abrigos filantrópicos e Oscips. Respectivamente, os primeiros são organizações filantrópicas e beneficentes voltadas para assistência social, sem fins lucrativos e em princípio da prática de caridade, não devendo ser confundidos com organizações não governamentais (ONGs), descritas como: sociedade civil, sem fins lucrativos, comprometidas com a transformação social. Já as Ocips são pessoas jurídicas de direito privado, sem fins lucrativos, constituídas no mínimo há três anos, funcionando regularmente, respeitando os requisitos determinados pela Lei no 9.790 (Brasil, 1999), e podem firmar parcerias com o poder público - federal, estadual ou municipal - para execução de atividades relacionadas a interesses públicos, como a assistência social (Brasil, 1999).

A escolha por abrigos dessa região ocorreu a partir do site Padrinho Nota 10 (2015), ONG que divulga uma lista de abrigos em todo o território nacional brasileiro, servindo de norteador para o levantamento de abrigos na Região Metropolitana de Campinas (SP). Constam na referida região aproximadamente trinta abrigos em funcionamento, segundo o site pesquisado, distribuídos entre as 20 cidades que a compõem. Foram realizadas pesquisas por meio do Conselho Municipal dos Direitos da Criança e do Adolescente (CMDCA) dos municípios que dispunham de abrigos infanto-juvenis, confirmando a preocupação em fornecer uma infraestrutura e políticas públicas que auxiliem crianças e adolescentes em situação de vulnerabilidade e suas famílias de origem nos programas oferecidos pelo poder público.

Aproximadamente nove abrigos apresentaram número para contato inexistente, dez não funcionavam em período integral e em torno de sete abrigos não aceitaram o convite para participar da pesquisa. Ao final, quatro abrigos institucionais estavam de acordo com os critérios de inclusão. A Tabela 1 demonstra as características dos quatro abrigos envolvidos neste estudo.

Tabela 1

Características dos abrigos institucionais infanto-juvenis $(\mathrm{N}=4)$.

\begin{tabular}{lcccc}
\hline & Abrigo A & Abrigo B & Abrigo C & Abrigo D \\
\hline Tipo de abrigo $^{\mathbf{a}}$ & Filantrópico & Filantrópico & Oscip $^{\mathrm{a}}$ & Oscip \\
Tempo de funcionamento (anos) & 30 & 39 & 16 & 37 \\
Número de abrigados & 18 & 17 & 36 & 48 \\
Número de abrigados com necessidades especiais & 01 & 0 & 0 & 0 \\
Faixa etária dos abrigados (anos) & $1-18$ & $12-18$ & $0-18$ & $0-18$ \\
Número de funcionários & 22 & 28 & 54 & 44 \\
Colaboradores externos & 08 & & & 15 \\
\hline
\end{tabular}

a Organizações da Sociedade Civil de Interesse Público 
Dos 63 profissionais dos quatro abrigos, 18 não preencheram os critérios de inclusão e, assim, a amostra final desta pesquisa envolveu 45 profissionais.

Dos 45 participantes, 24 são de abrigos filantrópicos e 21 de abrigos Oscips. Esses profissionais estavam distribuídos em quinze funções e para a realização das análises estatísticas descritivas, e foram divididos em dois grupos de acordo com suas funções: a) 34 participantes de contato direto com os abrigados: agente de proteção social, assistente social, coordenador, cozinheira, educador social, pedagoga, presidente, psicólogo; e b) 11 participantes de contato indireto com os abrigados: assistente administrativo, auxiliar de limpeza, marketing, motorista, pedreiro, recepcionista, supervisora.

Dos 34 participantes de contato direto com os abrigados, 58,8\% estavam em abrigos filantrópicos e $41,1 \%$ em abrigos Oscips, sendo $70,5 \%$ do gênero feminino e $29,4 \%$ do gênero masculino. A idade ficou entre 25 e 63 anos $(M=35,5)$. O estado civil casado e/ou união estável caracterizou $47 \%$ dos participantes, $35,2 \%$ eram solteiros, $8,8 \%$ divorciados e $8,8 \%$ elegeram a opção outros. Os níveis de escolaridade foram: superior completo com $38,2 \%$ dos participantes, $29,4 \%$ com superior incompleto, $17,6 \%$ com médio completo, $8,8 \%$ com médio incompleto, $2,9 \%$ com técnico completo e $2,9 \%$ com fundamental incompleto.

Em relação à dinâmica de trabalho, $47,0 \%$ dos participantes cumpriam uma jornada de trabalho de mais de 40 horas semanais, $32,3 \%$ trabalhava entre 31 e 40 horas semanais e $20,5 \%$ até 30 horas semanais. Em relação ao tempo que exerciam a função, $41,1 \%$ dos participantes estavam entre 7 meses e 2 anos, $17,6 \%$ de 2 a 5 anos, $38,2 \%$ acima de 5 anos, e 2,9\% participantes até 6 meses. Quanto ao tempo de trabalho no abrigo, $44,1 \%$ dos participantes estavam na faixa de 7 meses a 2 anos, $14,7 \%$ entre 2 e 5 anos, $32,3 \%$ acima de 5 anos, e 8,8\% até 6 meses de trabalho no abrigo.

O grupo de contato indireto com os abrigados incluiu 11 participantes, sendo $72,7 \%$ do gênero feminino e $27,2 \%$ do gênero masculino, com idade entre 29 e 63 anos $(M=45,0)$. O estado civil predominante foi casado e/ ou união estável, com $63,6 \%$ dos participantes, $18,1 \%$ eram solteiros, $9 \%$ eram divorciados e, na opção outro, $9,0 \%$ dos participantes. Os níveis de escolaridade encontrados foram: $27,2 \%$ com fundamental incompleto, $27,2 \%$ com superior completo,
$18,1 \%$ com fundamental completo, 9,0\% com médio incompleto, 9,0\% com médio completo e 9,0\% com superior incompleto.

Sobre a jornada de trabalho, $90,9 \%$ dos participantes cumpriam jornada de 31 a 40 horas semanais, e $9 \%$ dos participantes tinham jornada com mais de 40 horas semanais. O tempo na função descreve $54,5 \%$ com mais de 5 anos, $18,1 \%$ entre 2 e 5 anos, $18,1 \%$ entre 7 meses e 2 anos e $9,0 \%$ dos participantes com até 6 meses. Em relação ao tempo de trabalho no abrigo, os resultados indicaram que $36,3 \%$ dos participantes tinham mais de 5 anos, $36,3 \%$ entre 7 meses e 2 anos, 18,1\% com até 6 meses e 9,0\% dos participantes entre 2 e 5 anos no abrigo. Quanto ao tipo de abrigo, $36,3 \%$ dos participantes eram de abrigos filantrópicos e 63,6\% de abrigos Oscips.

\section{Instrumentos}

Foram utilizados quatro instrumentos: a) ficha de caracterização do abrigo institucional composta por questões referentes à estrutura física e da equipe profissional da instituição; b) ficha de caracterização do participante - questões referentes aos dados sociodemográficos dos participantes; c) Impact of Event Scale (IES) - criada por Horowitz, Wilner e Alvarez (1979) e adaptada por de Silva, Nardi e Horowitz (2010), para investigar o estresse relacionado aos eventos da vida, focando nas características que envolvam o evento, sem focar em uma situação especifica; d) Cope Breve - desenvolvido por Carver (1997) e adaptado por Brasileiro e Costa (2012), para avaliar as estratégias de coping em três subescalas: i) focalizado no problema (positivo); ii) focalizado na emoção e adaptativo (positivo com pequenas alterações); e iii) focalizado na emoção e desadaptativo (negativo, relacionado ao processo do adoecimento). O coping focalizado no problema tem como função alterar a dificuldade existente na relação entre as pessoas e o ambiente, podendo o indivíduo direcionar sua ação interna ou externamente. O coping focalizado na emoção e adaptativo permite a alteração do estado emocional do indivíduo, buscando reduzir e/ou adaptar a sensação física desagradável do estado de estresse e, finalmente, o coping focalizado na emoção e desadaptativo é a estratégia considerada mais prejudicial, pois não oferece possibilidades assertivas para o enfrentamento do evento estressor (Carver, Scheier, \& Weintraub, 1989; Lazarus \& Folkman, 1984). 


\section{Análises estatísticas}

Os dados obtidos foram organizados em um banco de dados, construído em Excel versão 2010 e, posteriormente, submetidos às análises estatísticas no software Statistical Package for the Social Sciences (SPSS), versão 20.

Para a análise dos resultados, utilizou-se a estatística descritiva valores máximo e mínimo das pontuações nas subescalas de coping, e também inferencial: análise de regressão linear e não paramétrico: correlação de Spearman, considerando a natureza e a distribuição dos escores. Na análise dos dados, foi utilizada a distribuição de participantes para caracterizar a amostra por meio das variáveis sociodemográficas.

$\mathrm{Na}$ análise do nível de estresse, os resultados foram determinados pelos escores totais da escala IES demonstrando o número de participantes em cada um desses níveis. Os estilos e/ou estratégias de coping utilizados pelos participantes foram analisados por meio da distribuição dos escores totais de cada subescala: coping focalizado no problema, coping focalizado na emoção e adaptativo e coping focalizado na emoção e desadaptativo.

Por meio da análise de regressão linear, foram descritas a variável dependente nível de estresse em função das variáveis independentes: tipo de abrigo, grupo de profissionais de contato direto e indireto com os abrigados (função), escolaridade, jornada, coping focalizado no problema, coping focalizado na emoção e adaptativo e coping focalizado na emoção e desadaptativo, com o objetivo de aprofundar a análise da relação entre essas variáveis. Para a análise de correlação entre estresse total e os estilos e/ou estratégias de coping, foi utilizado o coeficiente de correlação de Spearman, devido ao tamanho reduzido da amostra.

O nível de significância adotado para a regressão linear foi de $\leq 0,001$ e, para a correlação de Spearman, foram adotados os critérios de Dancey e Reidy (2004) para a intensidade do relacionamento e a magnitude, respectivamente: a) perfeito: 1 ; b) forte: $0,7-0,9$; c) moderado: 0,4-0,6; d) fraco: 0,1-0,3; e e) zero: 0 .

\section{Resultados}

Os resultados foram apresentados em níveis de estresse e os estilos e/ou estratégias de coping utilizados pelos participantes dos abrigos, regressão linear para os fatores preditores de estresse e as correlações entre o estresse e os de estilo e/ou estratégias de coping.

\section{Descrição dos níveis de estresse}

Por meio da escala IES, foram apurados os níveis de estresse por tipo de abrigo institucional e por grupo de participantes de contato direto e indireto com os abrigados, de acordo com o critério proposto por Silva et al. (2010).

Ao analisar os níveis de estresse, o grupo de participantes de contato direto com os abrigados em abrigo institucional filantrópico apresentou dois sujeitos no nível de 0-8 (não há evento estressor significativo); dois no nível de 9-25 (evento estressor de impacto), no qual o indivíduo pode ser afetado; sete no nível de 26-43 (evento estressor de impacto marcante), no qual o indivíduo será afetado; e nove no nível de 44-75 pontos (evento estressor de impacto grave), associado às alterações emocionais, psicológicas e físicas no indivíduo.

Tabela 2

Níveis de estresse por tipo de abrigo institucional e grupos de participantes $(\mathrm{N}=45)$.

\begin{tabular}{lcccc}
\hline & \multicolumn{4}{c}{ Nível de Estresse $^{\mathbf{a}}$} \\
\cline { 2 - 4 } & $0-8$ & $9-25$ & $26-43$ & $44-75$ \\
& $n$ & $n$ & $n$ & $n$ \\
\hline Participantes de contato direto com abrigados & & & \\
$\quad$ Abrigo Institucional Filantrópico & 2 & 2 & 7 & 9 \\
$\quad$ Abrigo Institucional OSCIP & 2 & 10 & 1 & 4 \\
Participantes de contato indireto com abrigados & & & \\
$\quad$ Abrigo Institucional Filantrópico & & & 2 & 1 \\
$\quad$ Abrigo Institucional OSCIP & 1 & 3 & 2 & \\
\hline
\end{tabular}

a0 - 8: não há evento estressor significativo; 9-25: evento estressor de impacto - pode ser afetado; 26 - 43: evento estressor de impacto marcante - será afetado; 44 - 75: evento estressor de impacto grave-pode causar alterações emocionais, psicológicas e físicas. 
Já o grupo de participantes de contato direto com os abrigados em abrigo Oscip apresentou dois participantes no nível de 0-8 pontos (não há evento estressor significativo); 10 no nível de 9-25 (evento estressor de impacto), no qual o indivíduo pode ser afetado; um no nível de 26-43 (evento estressor de impacto marcante), no qual o indivíduo será afetado; e um no nível de 44-75 pontos (evento estressor de impacto grave), associado a alterações emocionais, psicológicas e físicas no indivíduo.

O grupo de participantes de contato indireto com os abrigados apresentou quatro deles de abrigo institucional filantrópico no nível de 44-75 pontos (evento estressor de impacto grave), que está associado a alterações emocionais, psicológicas e físicas no indivíduo. O grupo de participantes de contato indireto com os abrigados de abrigo Oscip apresentou um participante no nível de 0-8, no qual não há evento estressor significativo; três no nível de 9-25 pontos, em que há evento estressor de impacto e no qual o indivíduo pode ser afetado; dois no nível de 26-43, no qual há evento estressordeimpactomarcanteeoindivíduoseráafetado, e um para o nível de 44-75 pontos, em que há evento estressor de impacto grave e está associado a alterações emocionais, psicológicas e físicas no indivíduo.

De modo geral, as maiores frequências se referem ao nível de estresse evento estressor de impacto em participantes que têm contato direto com os abrigados em abrigos Oscips $(\mathrm{n}=10)$, e nível de estresse evento estressor de impacto grave naqueles $(\mathrm{n}=9)$ que trabalham em contato direto com os abrigados, em abrigos filantrópicos.

\section{Descrição do estilo e/ou estratégia de coping mais utilizado pelos profissionais}

Para avaliar o coping, utilizou-se o inventário Cope Breve adaptado por Brasileiro e Costa (2012), que permitiu identificar os diferentes estilos e/ou estratégias de coping utilizados pelos participantes. A Tabela 3 demonstra o percentil, os escores mínimo e máximo dos estilos e/ou estratégias de coping apresentados pelos 45 participantes.

Tabela 3

Distribuição dos estilos e/ ou estratégias de coping para a amostra total $(\mathrm{N}=45)$.

\begin{tabular}{lcccc}
\hline Estilos e/ou estratégias de coping & $\boldsymbol{N}$ & $\boldsymbol{\%}$ & Mínimo & Máximo \\
\hline Focalizado no problema & 42 & 93,4 & 10 & 39 \\
Focalizado na emoção e adaptativo & 1 & 2,2 & 26 & 35 \\
Focalizado na emoção e desadaptativo & 2 & 4,4 & 22 & \\
\hline
\end{tabular}

A análise dos escores revelou que $93,4 \%$ dos participantes utilizaram o estilo e/ou estratégia coping focado no problema, considerado positivo, indicando que os participantes podem ter feito uso de estratégias de ação conjuntamente a um plano de ação, visando superar o estresse.

\section{Regressão linear para os fatores preditores de estresse}

Foi realizada a análise de regressão linear com o objetivo de determinar o efeito das variáveis: tipo de abrigo, grupo de profissionais de contato direto e indireto com os abrigados (função), escolaridade, jornada de trabalho, coping focalizado no problema, coping focalizado na emoção e adaptativo e coping focalizado na emoção e desadaptativo no nível de estresse.

A regressão linear mostrou que essas variáveis preveem o nível de estresse, pois o modelo foi estatisticamente significativo $\left(\mathrm{p}<0,001 ; \mathrm{R}^{2}=0,559\right)$. Assim, esse modelo explica 55,9\% da variância no nível de estresse e, a partir dele, pode-se verificar que o estilo de coping desadaptativo $(\beta=0,444 ; \mathrm{p}=0,005)$ é preditor do nível de estresse. Nesse sentido, à medida que o participante utilize mais o estilo e/ou estratégia de coping focalizado na emoção e desadaptivo (uma unidade), há uma previsão de aumento do seu estresse $(0,44$ unidade). A Tabela 4 apresenta o resultado da análise da regressão linear considerando as variáveis independentes selecionadas para a equação de regressão. 
Tabela 4

Regressão linear: variáveis preditoras do estresse.

\begin{tabular}{lcc}
\hline \multicolumn{1}{c}{ Variáveis } & Beta & p-valor \\
\hline $\begin{array}{l}\text { Coping focalizado na } \\
\text { emoção e desadaptativo }\end{array}$ & 0,444 & $<0,0001$ \\
Jornada de trabalho & $-0,114$ & 0,030 \\
Escolaridade & $-0,215$ & 0,057 \\
Tipo Abrigo & $-0,289$ & 0,0009 \\
\hline
\end{tabular}

asignificância.

Nota-se que o tipo de abrigo também foi uma variável que previu o nível de estresse, porém de modo negativo, ou seja, ao diminuir uma unidade, a tendência é aumentar 0,289 unidade na medida do estresse.

Das sete variáveis incluídas na análise, três não contribuíram para a explicação da variação do estresse: tipo de função, coping focado no problema e coping focado na emoção e adaptativo.

\section{Correlações entre o estresse e os de estilo e/ou estratégia de coping}

A análise de correlação foi realizada pelo coeficiente de correlação de Spearman, devido ao número amostral desta investigação. Os coeficientes de correlação obtidos entre os estilos e/ou estratégias de coping e o estresse mostram com que intensidade essas variáveis se associam. Nesse sentido, as associações foram analisadas tanto em relação à intensidade e direção da correlação quanto à sua significância estatística, adotando os critérios de Dancey e Reidy (2004). A Tabela 5 mostra as correlações entre estilos e/ou estratégias de coping e estresse.

Tabela 5

Correlação de Spearman entre estilos e/ou estratégias de coping e estresse $(\mathrm{N}=45)$.

\begin{tabular}{lc}
\hline \multicolumn{1}{c}{ Estilos de Coping } & Estresse \\
\hline Focalizado no problema & $0,363^{*}$ \\
$\begin{array}{l}\text { Focalizado na emoção e } \\
\text { adaptativo }\end{array}$ & 0,284 \\
$\begin{array}{l}\text { Focalizado na emoção e } \\
\text { desadaptativo }\end{array}$ & $0,598^{* *}$ \\
\hline
\end{tabular}

${ }^{*} p \leq 0,05 ;{ }^{* *} p \leq 0,001$.

Pode-se observar, a partir da Tabela 5, que houve correlação estatisticamente significativa entre dois estilos de coping e estresse. Essas associações foram positivas, indicando que, à medida que aumenta o nível de estresse, há uma tendência de aumentar as estratégias de coping, sendo que o coping focalizado na emoção e desadaptativo tende a aumentar mais do que o coping focalizado no problema, devido à intensidade da sua associação com o estresse ser maior (Dancey \& Reidy, 2004). O coping focalizado na emoção e adaptativo não se associou significativamente ao estresse.

Os resultados indicam que a correlação existe, mas somente em dois estilos de coping ela se mostrou significativa, apontando que hipótese nula foi refutada. Todas as correlações apresentadas foram positivas, mostrando que ambas as variáveis analisadas aumentam ou diminuem conforme a variação da outra, isto é, se o coping aumenta, o estresse também aumenta.

\section{Discussão}

O objetivo deste estudo foi verificar e analisar os níveis de estresse percebido e o estilo e/ou estratégia de coping dos profissionais das equipes de abrigos institucionais infanto-juvenis. Para alcançar esses objetivos, foram utilizados quatro instrumentos: ficha de caracterização do abrigo institucional, ficha de caracterização do participante, a escala Impact of Event Scale (IES) e o inventário Cope Breve.

Os instrumentos IES e o Cope Breve foram validados e testados em diferentes populações e apresentaram resultados confiáveis para utilização, indicando que os parâmetros metodológicos de adaptação para a língua portuguesa são recomendados para esse fim. Dessa maneira, os instrumentos escolhidos para este estudo já foram adaptados e utilizados no contexto brasileiro em pesquisas relacionadas à avaliação de estresse pós-traumático (Magalhães et al., 2018; Silva et al., 2010); pacientes portadores de transtorno bipolar (KluweSchiavon, Fabres, Daruy-Filho, \& Grassi-Oliveira, 2011); na odontopedriatria, com responsáveis por crianças em atendimento (Brasileiro \& Costa, 2012); com mulheres em tratamento por endometriose (Donatti, Ramos, Andres, Passman, \& Podgaec, 2017); e em outros países, como Portugal, com pessoas soropositivas ao vírus da aids/HIV (Duque, Reis, Lencastre, \& Guerra, 2017) e pacientes vítimas de lesão vertebro-medular traumática (Teixeira, Alves, Santos, \& Gestal-Otero, 2014).

Os 45 profissionais que participaram deste estudo estavam distribuídos em quatro abrigos institucionais localizados na Região Metropolitana de Campinas (SP), sendo dois filantrópicos e dois do tipo 
Oscip (Tabela 1). A composição da equipe de profissionais, regulamentada pela Resolução no 130 , de 2005 do CNAS, e as características internas conforme as Orientações técnicas: Serviços de acolhimento para crianças e adolescentes (Resolução no 109, 2009) foram respeitadas pelos quatro abrigos institucionais.

A partir das análises dos dados sociodemográficos, foi traçado o perfil dos profissionais dos abrigos institucionais. Em sua maioria, esses profissionais são do gênero feminino (71\%), na faixa de 39 anos de idade, casadas e/ou em união estável, com filhos e escolaridade superior completa ou incompleta. A jornada de trabalho predominante foi de 31 a 40 horas semanais, o tempo que desempenhavam suas funções foi, em média, seis anos, a maioria $(53,3 \%)$ trabalhava diretamente com os abrigados e o tempo de trabalho no abrigo foi, em média, de quatro anos.

No que se referiu à amostra masculina, $28,8 \%$ dos profissionais eram do gênero masculino, com idade média de 40 anos, em sua maioria casados e/ou em união estável e o nível de escolaridade predominante foi o superior incompleto, cumprindo jornada de trabalho de acima de 40 horas semanais.

Para Moreira (2016), esse perfil indica que, ainda hoje, o abrigamento institucional associa a figura feminina ao cuidado, principalmente em abrigos que atendam bebês e crianças pequenas. E, em abrigos que recebem adolescentes, a figura masculina se associa à imagem de uma postura um pouco mais rígida para a imposição de limites, perpetuando estereótipos referentes às figuras feminina e masculina na sociedade (Cavalcante \& Corrêa, 2012).

Neste estudo, um abrigo se destacou em relação a essa questão com a função de agente de proteção social, que tem como atividade acompanhar os adolescentes em atividades fora do abrigo, em que é necessário ter atenção e controle para que os abrigados não deixem de realizar ou se dispersem dos objetivos propostos dessas saídas do abrigo.

A questão sobre a escolaridade indicou que mais da metade dos participantes tem escolaridade acima da exigida para as funções executadas. Observou-se que muitos participantes na função de educador/ cuidador têm formação acadêmica em Psicologia, Serviço Social e Pedagogia. As orientações técnicas do Conanda (Brasil, 2009b) sobre essa função determinam como escolaridade mínima o ensino médio completo, com capacitação específica em cuidados básicos de crianças e adolescentes, não existindo uma formação acadêmica específica exigida para essa função.
No entanto somente o nível de escolaridade não prepara os profissionais para as necessidades específicas das crianças, dos adolescentes e de suas famílias de origem. Os profissionais citados anteriormente estão diariamente em contato direto com os abrigados, demonstrando a importância da capacitação continuada no contexto de abrigamento para o desenvolvimento de uma prática adequada, além do conhecimento sobre o ECA, programas de auxílio, políticas públicas, drogas, sexualidade, desenvolvimento humano, entre outros assuntos pertinentes a esse contexto, e que devem ser atualizados constantemente (Brito \& Souza, 2011).

Para Bazon e Biasoli-Alves (2000), os cursos de capacitação deveriam ser incentivados, assim como espaços para troca de experiências entre os profissionais, no entanto, a maioria dos cursos, palestras e eventos relacionados à capacitação para esses profissionais ocorre durante o horário de trabalho, o que pode se configurar como uma situação impeditiva para a participação desses profissionais. Ainda assim, é necessário que os profissionais participem de cursos de capacitação e busquem conhecimento para suas práticas.

Neste estudo, pôde-se analisar o aspecto da rotatividade de profissionais em abrigos institucionais. Os participantes trabalham nos abrigos, em média, há quatro anos, tempo que não pode ser considerado como de grande rotatividade. Mesmo essa questão sendo analisada em relação aos educadores/cuidadores, a média apresentada foi de dois anos e seis meses no mesmo abrigo e mesma função, não confirmando a maioria dos achados na literatura consultada sobre a rotatividade nesse contexto (Almeida, 2016; Poletto, Koller \& Dell'Aglio, 2009).

A baixa rotatividade de profissionais nos abrigos participantes colabora com a comunicação entre os profissionais, com o desenvolvimento adequado do Plano Individual de Atendimento (PIA), no planejamento de atividades e ações. Há também a questão da estabilidade financeira e profissional, que promove a segurança para o exercício profissional. Em relação às crianças e adolescentes abrigados, essa baixa rotatividade pode trazer um sentimento de segurança e pode proporcionar a formação de vínculo com o profissional, visto que estão afastadas de suas famílias de origem e necessitam de cuidados e atenção. O serviço de abrigamento não deve substituir a família de origem, mas os profissionais são incentivados a contribuir para um ambiente familiar no qual as necessidades 
específicas de cada um dos abrigados devem ser supridas da melhor maneira. A baixa rotatividade dos abrigos deste estudo demonstra a possibilidade de práticas continuadas que favorecem a todos os envolvidos (Cavalcante \& Corrêa, 2012; Moreira, 2016).

Tendo em vista que o contexto de abrigos institucionais infanto-juvenis exige dos profissionais certa urgência nas resoluções e no atendimento das necessidades das crianças, adolescentes e suas famílias, o contato diário direto ou indireto com esses em suas práticas, somado à jornada de trabalho e à vida pessoal, podem ser considerados eventos estressores. Para analisar e compreender o nível de estresse em que se encontram os profissionais de abrigos, utilizaram-se como referencial os níveis de estresse proposto por Horowitz et al. (1979) na escala IES.

Os resultados indicaram que os maiores escores se referem ao nível de estresse evento estressor de impacto em participantes que têm contato direto com os abrigados em abrigos Oscips $(\mathrm{n}=10)$, e nível de estresse evento estressor de impacto grave naqueles $(n=9)$ que trabalham em contato direto com os abrigados em abrigos filantrópicos (Tabela 2).

Esses escores demonstram que o grupo de contato direto de abrigos Oscips está na chamada fase de resistência, na qual há um desgaste do organismo por tentar restabelecer o equilíbrio interno, resistindo ao fator estressante, utilizando de mecanismos para controlar o estresse. E o grupo de contato direto de abrigos filantrópicos está na chamada fase de exaustão, associada ao processo de adoecimento, com sintomas de alteração de sono, irritabilidade, perda de concentração e desenvolvimento de doenças cardiovasculares, úlceras gástricas e depressão, segundo Lipp (2002).

Para Margis, Picon, Cosner e Silveira (2003), se as respostas aos eventos estressores forem ativadas constantemente e com intensidade, o organismo pode sofrer um esgotamento de recursos e, consequentemente, o nível de estresse pode aumentar, causando maiores prejuízos ao profissional. Neste estudo, observou-se também que profissionais de contato direto de abrigos filantrópicos $(\mathrm{n}=7)$ se alocaram no nível de estresse evento estressor de impacto marcante, denominado por Lipp (2002) de fase de quase exaustão, na qual o organismo teria dificuldades para se adaptar aos eventos estressores, sem conseguir gerenciar a tensão e realizar as atividades diárias. Assim, esses profissionais correm o risco de elevar seu nível de estresse.
A função do cuidar para autores como Camelo e Angerami (2004) e Lima (2012) pode ser considerada um fator estressor devido a responsabilidades, urgências nas resoluções de problemas, excesso de preocupações e de tarefas, resultando em insatisfação, esgotamento emocional, que pode prejudicar o exercício da função, interferindo nas resoluções diárias e nas demandas dos abrigados a serem atendidas. O processo de estresse será compreendido, desencadeado e desenvolvido de maneiras diferentes para cada profissional, de acordo com suas vivências, experiências e subjetividade. Consequentemente, as respostas e os esforços adaptativos também serão distintos.

Para explicar os estilos e/ou estratégias de coping, serão discutidos os escores a partir das três subescalas de Cope: a) focalizado no problema considerado positivo; b) focalizado na emoção e adaptativo - considerado positivo, podendo afetar a ação; e c) focalizado na emoção e desadaptativo - considerado negativo. Buscou-se, dessa maneira, identificar os estilos e/ou estratégias mais utilizados pelos profissionais de abrigos (Brasileiro \& Costa, 2012; Carver, 1997).

Neste estudo, o estilo e/ou estratégia de coping mais utilizado pelos participantes $(n=42)$ foi o focado no problema e, ao dividir os grupos de participantes em contato direto e indireto com os abrigados e por tipos de abrigos filantrópicos e Oscips, o coping focalizado no problema permanece como o mais utilizado (Tabela 3). Para Antoniazzi, Dell'Aglio e Bandeira (1998), a função desse estilo e/ou estratégia de coping seria a de alterar o evento estressor existente, na situação que deu início à tensão, buscando alterá-la, o que justificaria os níveis de estresse apresentados pelos participantes: evento estressor de impacto $(n=10)$ e evento estressor de impacto grave $(n=9)$.

$\mathrm{Na}$ análise das variáveis preditoras do estresse (Tabela 4), quatro variáveis - coping focalizado na emoção e desadaptativo $(p \leq 0,0001)$, tipos de abrigos ( $p=0,0009)$, jornada de trabalho $(p=0,030)$ e escolaridade $(p=0,057)$ - foram responsáveis por $69,96 \%$ da variância no estresse $\left(\mathrm{R}^{2}\right)$. Foi verificado que o coping focalizado na emoção e desadaptativo e os tipos de abrigos apresentaram maior significância estatística, demonstrando ser os preditores mais significativos para o estresse, isto é, um nível de estresse mais elevado poderia estar diretamente relacionado a essas variáveis.

Quando se fala de abrigo como um preditor de estresse, fala-se das condições oferecidas para os profissionais exercerem suas práticas, tais como 
infraestrutura, remuneração, jornada de trabalho, quantidade adequada de profissionais para desenvolver atividades, incentivos a capacitação continuada, fatores que podem ser considerados como eventos estressores, e serão compreendidos e interpretados pelos profissionais de acordo com a percepção de cada um deles.

A preocupação com o bem-estar das crianças e dos adolescentes sob seus cuidados, as necessidades de cada abrigado e, por vezes, de sua família, o excesso de atividades a ser desempenhada pelos profissionais dentro e fora do abrigo, a carga horária semanal, as cobranças constantes por parte da instituição, além das exigências pessoais, revelam que esses profissionais podem apresentar alterações físicas e psíquicas durante sua jornada de trabalho. Para Cavalcante e Corrêa (2012) e Moreira (2016), essa proximidade com os abrigados, diariamente, pode causar vulnerabilidade emocional nos profissionais e pode afetar suas práticas em relação a esses.

Já autores como Bazon e Biasoli-Alves (2000) explicam que o ideal é que os profissionais deem sentido à sua prática, procurando perceber o real objetivo do seu trabalho e como este pode ou não influenciar os abrigados. Na maioria das vezes, durante sua jornada de trabalho, esses profissionais não dispõem de uma infraestrutura para troca de conhecimento, exposição dos desafios enfrentados diariamente e acabam contando somente com seus próprios recursos para executar seu trabalho.

Por essa razão, a escolaridade também ser um dos preditores do estresse é consoante com o que foi exposto sobre os dois primeiros preditores: abrigo e jornada de trabalho. Neste estudo, $60 \%$ dos participantes têm nível superior, seja completo ou incompleto, indicando que dispõem de qualificação para desenvolver as atividades referentes a suas funções. No entanto somente a capacitação não prepara os profissionais para o dia a dia do abrigo - a experiência e as habilidades adquiridas no decorrer da prática é que podem trazer subsídios para que esses profissionais lidem com os eventos estressores e utilizem estratégias de enfrentamento adequadas.

Para Almeida (2016) e Cavalcante e Corrêa (2012), as orientações técnicas (Resolução no 109,2009 ) ressaltam a importância da educação, da capacitação continuada para os profissionais de abrigos institucionais, seja por meio de cursos, seminários, palestras ou treinamentos específicos, principalmente para os profissionais de contato direto com os abrigados, que ficam expostos diariamente às demandas desses e às exigências dos abrigos. A aquisição de conhecimento adequado para as demandas diárias dos profissionais é de suma importância. Apesar disso, na maioria das vezes, a jornada de trabalho não permite que haja tempo para a capacitação e os abrigos nem sempre conseguem liberar seus profissionais durante o expediente para que participem de cursos.

Nessa perspectiva, Cavalcante e Corrêa (2012) e Dias (2016) consideram que são exigidas dos profissionais cargas afetivas, cognitivas e físicas consideradas excessivas, que podem levar a um desgaste acumulado e, consequentemente, ao adoecimento. No caso deste estudo, levaria ao aumento do nível de estresse.

Considerando o exposto, o coping focalizado na emoção e desadaptativo ter apresentado maior significância estatística e uma relação positiva com o estresse contribui com a ideia de que nem sempre o que está prescrito nas normas e nos manuais de orientação para as práticas profissionais é possível de ser colocado em prática no cotidiano. Autores como Halpern, Leite e Moraes (2015) afirmam haver uma linha tênue entre a realidade profissional e a pessoal, e, devido a esse fato, nem sempre os profissionais estão preparados para lidar com as demandas dos abrigados e controlar os sentimentos que surgem desse contato tão próximo.

O coping focalizado na emoção e desadaptativo ser um preditor do estresse demonstra que os profissionais de abrigos podem negar a existência dos estressores, buscam alternativas para afastar da mente, evitam ter contato e, quando essas estratégias não são suficientes, a tendência à autocrítica e ao uso de substâncias psicoativas pode ser uma alternativa. Esse estilo e/ou estratégia de coping pode ser considerado o mais prejudicial para os profissionais, visto que pode causar prejuízos a eles mesmos ou para outros - em nosso caso, às crianças e aos adolescentes abrigados.

A última análise a ser discutida é a correlação de Spearman entre estilos e/ou estratégias de coping e estresse. Constatou-se que todos os estilos e/ou estratégias de coping se correlacionam com o estresse positivamente, entretanto o coping focalizado na emoção e desadaptativo apresenta uma significância estatística maior com o estresse (Tabela 5).

Os valores das correlações não foram muito fortes: a) o coping focalizado no problema, quando correlacionado com estresse, apresentou correlação 
fraca; b) o coping focalizado na emoção e desadaptativo, quando correlacionado com estresse, apresentou correlação moderada; e c) no coping focalizado na emoção e adaptativo, a correlação foi considerada insignificante $(<0,3)$.

Os resultados obtidos demonstram a importância dos profissionais de abrigos se capacitarem e disporem de espaço para que possam ser ouvidos, expor experiências e falar sobre o dia a dia com os abrigados. Por meio das análises, constatou-se também que alguns profissionais dos abrigos institucionais necessitam de auxílio para compreender o que ocorre em seu dia a dia e como podem lidar ou não com os eventos estressores, devido ao alto nível de estresse (evento estressor de impacto grave).

\section{Considerações finais}

Este estudo objetivou verificar os níveis de estresse percebido e os estilos e/ou estratégias de coping dos profissionais das equipes de abrigos institucionais infanto-juvenis. A maior dificuldade na consecução do objetivo foi a escassez de estudos relativos a essa população e, por conseguinte, a interlocução com a literatura da área.

Contudo os resultados se mostraram significativos para responder às questões da pesquisa e aumentar o conhecimento sobre o tema. As conclusões elencadas a seguir traduzem essa contribuição:
- a maioria dos participantes foi do gênero feminino;

- os níveis de estresse percebidos foram maiores nos participantes que têm contato direto com os abrigados, em abrigos institucionais infantojuvenis filantrópicos;

- as estratégias de coping mais utilizadas tanto pelos participantes de abrigos filantrópicos quanto pelos de abrigos Oscips foi o coping focalizado no problema;

- as variáveis preditoras de estresse foram o tipo de abrigo, a jornada de trabalho, a escolaridade e o coping focalizado na emoção e desadaptativo;

- entre os estilos de coping, aquele com maior correlação com o estresse foi o focalizado na emoção e desadaptativo.

A partir dessas conclusões, acredita-se na possibilidade e necessidade de estudos futuros que se debrucem sobre as condições de saúde dessa população, pesquisando as funções, de modo específico, e suas relações com os diferentes tipos de abrigos.

Somente a avaliação da saúde dos profissionais de abrigos institucionais infanto-juvenis filantrópicos e Oscips poderá gerar ações e políticas adequadas para detectar as necessidades de aprimoramento e/ou programas de capacitação profissional, contribuindo, em última análise, para a qualidade da assistência às crianças e adolescentes abrigados.

\section{Referencias}

Almeida, V. P. (2016). Formação e capacitação dos profissionais das casas de acolhimento institucional de crianças e adolescentes: As oficinas de dinâmica de grupo coconstruindo saberes. In M. I. C. Moreira, A. P. C. P. Passos, \& V. P. Almeida (Orgs.), Cuidar de quem cuida: Trabalhadoras e trabalhadores no acolhimento institucional (pp 67-94). CRV.

Antoniazzi, A. S., Dell'Aglio, D. D., \& Bandeira, D. R. (1998). O conceito de coping: Uma revisão teórica. Estudos de Psicologia, 3(2), 273-294. https://doi.org/10.1590/S1413294X1998000200006

Barros, N. S. (2014). Capacitação para educadores de abrigo de crianças e adolescentes: Identificando representações sociais [Dissertação de mestrado, Universidade Federal Rural do Rio de Janeiro]. Biblioteca Digital de Teses e Dissertações da UFRRJ. https://tede.ufrrj.br/jspui/handle/jspui/3369

Bazon, M. R., \& Biasoli-Alves, Z. M. M. (2000). A transformação de monitores em educadores: Uma questão de desenvolvimento. Psicologia: Reflexão e Crítica, 13(1), 199-204. https://doi.org/10.1590/S0102-79722000000100020

Brasileiro, S. V., \& Costa, L. R. R. S. (2012). Adaptação transcultural e propriedades psicométricas do Cope Breve em uma amostra brasileira [Dissertação de mestrado, Universidade Federal de Goiás]. Biblioteca Digital de Teses e Dissertações da UFG. https://repositorio.bc.ufg.br/tede/handle/tede/3351

Brito, C. V., \& Souza, J. C. (2011). Qualidade de vida dos educadores sociais em abrigos de proteção a crianças e adolescentes. Psicologia: Teoria e Prática, 13(1), 89-100.

Camelo, S. H. H., \& Angerami, E. L. S. (2004). Sintomas de estresse nos trabalhadores atuantes em cinco núcleos de saúde da família. Revista Latino-Americana de Enfermagem, 12(1), 14-21. https://doi.org/10.1590/ S0104-11692004000100003 
Carver, C. (1997). You want to measure coping but your protocol's too long: Consider the brief COPE. International Journal of Behavioral Medicine, 4(92). https://doi.org/10.1207/s15327558ijbm0401_6

Carver, C. S, Scheier, M. F., \& Weintraub, J. K. (1989). Assessing coping strategies: A theoretically based approach. Journal of Personality and Social Psychology, 56, 267-283.

Cavalcante, L. I. C., \& Corrêa, L. S. (2012). Perfil e trajetória de educadores em instituição de acolhimento infantil. Cadernos de Pesquisa, 42(146), 494-517. https://doi.org/10.1590/S0100-15742012000200010

Dancey, C. P., \& Reidy, J. (2004). Estatística sem matemática para psicologia: Usando SPSS para Windows (3a ed.). ArtMed.

Dias, M. D. A. (2016). A saúde do trabalhador da casa de acolhimento institucional. In M. I. C. Moreira, A. P. C. P. Passos, \& V. P. Almeida (Orgs.), Cuidar de quem cuida: Trabalhadoras e trabalhadores no acolhimento institucional (pp. 95-112). CRV.

Donatti, L., Ramos, D. G., Andres, M. P., Passman, L. J., \& Podgaec, S. (2017). Pacientes com endometriose que utilizam estratégias positivas de enfrentamento apresentam menos depressão, estresse e dor pélvica. Einstein (São Paulo), 15(1), 65-70. https://doi.org/10.1590/s1679-45082017ao3911

Duque, S., Reis, A. C., Lencastre, L. Q., \& Guerra, M. P. (2017). Satisfação com a vida em pessoas soropositivas ao vírus da SIDA. Análise Psicológica, 35(3), 297-308. https://doi.org/10.14417/ap.1183

Estado de São Paulo. (2000). Lei Complementar Estadual no 870, de 19 de junho de 2000. https:/ /www.al.sp.gov.br/ norma/?id=5198\#: :text=Cria\%20a\%20Regi\%C3\%A3o\%20Metropolitana\%20de,Campinas\%2C\%20e\%20d\% C3\%A1\%20provid\%C3\%AAncias\%20correlatas.

Guará, I. M. F. R. (2010). Abrigo: Comunidade de acolhida e socioeducação. In M. V. Baptista (Coord.), Abrigo: Comunidade de acolhida e socioeducação (2a ed., pp. 59-72). Instituto Camargo Corrêa.

Halpern, E.E., Leite, L. M. C., \&Moraes, M. C. M. B. (2015). Seleção, capacitação e formação da equipe de profissionais dos abrigos: O hiato entre o prescrito e o real. Trabalho, Educação e Saúde, 13(Suppl.1), 91-113. https://doi.org/ 10.1590/1981-7746-sip00033

Horowitz, M., Wilner, N., \& Alvarez, W. (1979). Impact of event scale: A measure of subjective stress. Psychosomatic Medicine, 41, 209-218.

Kluwe-Schiavon, B., Fabres, C. S., Daruy-Filho, L., \& Grassi-Oliveira, R. (2011, 3-7 outubro). Adequação da Brief COPE como medida de coping traço [Artigo Apresentado]. Anais do $12^{\circ}$ Salão de Iniciação Científica PUCRS, Porto Alegre, RS, Brasil.

Lazarus, R. S., \& Folkman, S. (1984). Stress, appraisal, and coping. Springer.

Lei no 8.069, de 13 de julho de 1990, Brasil (1990). Dispõe sobre o Estatuto da Criança e do Adolescente e dá outras providências. http://www.planalto.gov.br/ccivil_03/leis/L8069.htm

Lei no 9.790, de 23 de março de 1999, Brasil. (1999). Dispõe sobre a qualificação de pessoas jurídicas de direito privado, sem fins lucrativos, como Organizações da Sociedade Civil de Interesse Público, institui e disciplina o Termo de Parceria, e dá outras providências. http://www.planalto.gov.br/Ccivil_03/LEIS/L9790.htm

Lei $n^{\circ} 12.010$, de 3 de agosto de 2009, Brasil (2009). Dispõe sobre adoção; altera as Leis $n^{\text {os }} 8.069$, de 13 de julho de 1990 - Estatuto da Criança e do Adolescente, 8.560, de 29 de dezembro de 1992; revoga dispositivos da Lei $\mathrm{n}^{\circ}$ 10.406, de 10 de janeiro de 2002 - Código Civil, e da Consolidação das Leis do Trabalho - CLT, aprovada pelo Decreto-Lei $\mathrm{n}^{\circ}$ 5.452, de $1^{\circ}$ de maio de 1943; e dá outras providências. http://www.planalto.gov.br/ ccivil_03/_ato2007-2010/2009/lei/112010.htm

Lima, S. C. C. (2012). O trabalho do cuidado: Uma análise psicodinâmica. Revista Psicologia Organizações e Trabalho, 12(2), 203-215.

Lipp, M. E. N. (2000). O stress está dentro de você (2a ed.). Contexto.

Lipp, M. E. N. (2002). O estresse do professor. Papirus.

Magalhães, S. S., Miranda, D. K., Paula, J. J., Miranda, D. M., Romano-Silva, M. A., \& Malloy-Diniz, L. F. (2018). Psychometric properties of a Brazilian Portuguese version of theChildren's Revised Impact of Event Scale (CRIES-8). Archives of Clinical Psychiatry, 45(2), 27-32. https://doi.org/10.1590/0101-60830000000151

Margis, R., Picon, P., Cosner, A. C., \& Silveira, R. O. (2003). Relação entre estressores, estresse e ansiedade. Revista de Psiquiatria do Rio Grande do Sul, 25(Suppl.1), 65-74. https://doi.org/10.1590/S0101-81082003000400008

Moreira, M. I. C. (2016). Trabalhadoras e trabalhadores nas casas de acolhimento institucional: Diagnóstico e perspectivas. In M. I. C. Moreira, A. P. C. P. Passos, \& V. P. Almeida (Orgs.), Cuidar de quem cuida: Trabalhadoras e trabalhadores no acolhimento institucional (pp. 15-42). CRV. 
Norma Operacional Básica de Recursos Humanos do SUAS, Brasil (2006). Ministério do Desenvolvimento Social e Combate à Fome, Secretária Nacional de Assistência Social Sistema Único de assistência Social. http://www.assistenciasocial.al.gov.br/sala-de-imprensa/arquivos/NOB-RH.pdf/view

Orientações técnicas: Serviços de acolhimento para crianças e adolescentes, Brasil (2009b). Ministério do Desenvolvimento Social e Combate à Fome. http://www.mds.gov.br/webarquivos/publicacao/ assistencia_social/Cadernos/orientacoes-tecnicas-servicos-de-alcolhimento.pdf

Padrinho Nota 10. (2015). Lista de orfanatos e abrigos. http://www.padrinhonota10.com.br/default.asp? $\mathrm{Pag}=1 \&$ Destino $=$ Instituicoes\&Estado=SP

Poletto, M., Koller, S. H., \& Dell'Aglio, D. D. (2009). Eventos estressores em crianças e adolescentes em situação de vulnerabilidade social de Porto Alegre. Ciência \& Saúde Coletiva, 14(2), 455-466. https://doi.org/10.1590/ S1413-81232009000200014

Selye, H. (1956). Stress: A tensão da vida. Ibrasa.

Simões, R. L. (2012). Estratégias de coping em profissionais de uma instituição de privação de liberdade para adolescentes em conflito com a lei [Dissertação de mestrado, Universidade de São Paulo]. Biblioteca Digital de Teses e Dissertações da USP. https:// doi.org/10.11606/D.22.2012.tde-16012013-102501

Silva, A. C. O., Nardi, A. E., \& Horowitz, M. (2010). Versão brasileira da Impact of Event Scale (IES): Tradução e adaptação transcultural. Revista Psiquiatria Rio Grande do Sul, 32(3), 86-93. https://doi.org/10.1590/ S0101-81082010000300005

Silva, A. M. B., \& Enumo, S. R. F. (2014). Estresse, autorregulação e estratégias de enfrentamento [Artigo Apresentado].

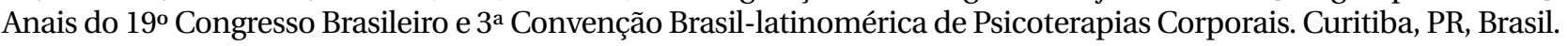

Siqueira, A. C., Betts, M. K., \& Dell'Aglio, D. D. (2006). A rede de apoio social e afetivo de adolescentes institucionalizados no Sul do Brasil. Internamerican Journal of Psychology, 40(2), 149-158. http://www.redalyc.org/ articulo.oa?id=28440202

Teixeira, A. R., Alves, J. B., Santos, A., \& Gestal-Otero, J. (2014). Lesão medular traumática: Impacto das variáveis sociodemográficas no ajustamento e saúde mental dos sujeitos afectados. Psicologia, Saúde \& Doenças, 15(1), 110-120. https://doi.org/10.15309/14psd150110

\section{Rosângela Maria Negri Ferrão Pagnota}

Mestranda em Psicologia na Pontifícia Universidade Católica de Campinas (PUC-Campinas), Campinas -SP. Brasil.

E-mail: rosangela.negri@yahoo.com.br

(D) https://orcid.org/0000-0002-3780-3689

\section{Helena Bazanelli Prebianchi}

Docente do Programa de Pós-Graduação em Psicologia da PUC-Campinas, Campinas - SP. Brasil.

E-mail: helenaprebianchi@gmail.com

(1) https://orcid.org/0000-0002-3838-8413

Endereço para envio de correspondência:

Pontifícia Universidade Católica de Campinas (PUCC). Av. John Boyd Dunlop, s/n, Instituto de Psicologia e

Fonoaudiologia, Jardim Ipaussurama. CEP:13060-904. Campinas - SP. Brasil.

Recebido 20/03/2018

Aceito 19/03/2019

Received $03 / 20 / 2018$

Approved 03/19/2019

Recibido 20/03/2018

Aceptado 19/03/2019 
Como citar: Pagnota, R. M. N. F., \& Prebianchi, H. B. (2021). Estresse e estratégias de enfrentamento em profissionais de abrigos institucionais. Psicologia: Ciência e Profissão, 41, 1-15. https://doi.org/10.1590/1982-3703003193180

How to cite: Pagnota, R. M. N. F., \& Prebianchi, H. B. (2021). Stress and coping strategies in shelter professionals. Psicologia: Ciência e Profissão, 41, 1-15. https://doi.org/10.1590/1982-3703003193180

Cómo citar: Pagnota, R. M. N. F., \& Prebianchi, H. B. (2021). Estrés y estrategias de enfrentamiento de profesionales de refugios institucionales. Psicologia: Ciência e Profissão, 41, 1-15. https://doi.org/10.1590/1982-3703003193180 